\title{
Nanotecnología, patentes y la situación en América Latina
}

\author{
Homero F. Pastrana*, Alba Ávila**, \\ Germán Moreno***
}

\begin{abstract}
RESUMEN: El presente documento realiza una revisión del estado de las patentes en nanotecnología en las tres principales oficinas de patentes del mundo: USPTO, EPO, WIPO; evidenciando su comportamiento y los países más activos desde el año 1976 hasta el primer semestre de 2012. Se identifican también las patentes en nanotecnología generada desde Argentina, Brasil Chile y Colombia, con las interacciones de estas naciones entre sí y el efecto de la participación de la academia en la generación de dichas patentes.

Finalmente, se describe el proceso de solicitud de patentes en uno de los países miembros del tratado de cooperación en patentes PCT y resultados exitosos de empresas que trabajan en nanotecnología en la región.
\end{abstract}

\section{IMPORTANCIA DE SU DIVULGACIÓN}

Los resultados de la investigación aplicada llegan a su culminación al obtenerse patentes por el desarrollo. Resulta relevante en la región crear conciencia de la importancia en la generación de desarrollos patentables para los gobiernos, academia e investigadores. Creando ambientes favorables donde interacciones en conjunto con la industria.

\section{INTRODUCCIÓN}

Las patentes son indicadores del desarrollo de una sociedad al llevar a aplicación sus trabajos en investigación. Al mismo tiempo, permiten medir los resultados para la incorporación de nuevas tecnologías en la sociedad. En el caso de la nanotecnología, ésta ha dado auge a un sinnúmero de potenciales ideas innovadoras, los reportes en que se describen sus ventajas son comúnmente conocidos. Sin embargo, la transición de innovación a propiedad sobre ella no ha avanzado en la misma proporción (WIPO, 2012b).

Para que las actividades de investigación culminen en patentes es fundamental que los productos del desarrollo tengan la capacidad de beneficiar a la sociedad a

* Estudiante Doctoral. Facultad de IngenieríaEléctrica y Electrónica, Universidad de Los Andes.Cra1 № 18A12 Bogotá, (Colombia) Tels: +5713394949 Ext 2828 Fax: +571 3394999. hf.pastrana122@uniandes.edu.co

** Profesor Asociado. Facultad de Ingeniería Eléctrica y Electrónica, Universidad de Los Andes. Cra 1 № 18A12 Bogotá, (Colombia) Tels: +5713394949 Ext 2828 Fax: +571 3394999. a-avila@uniandes.edu.co

*** Gerente General, Kemtek@. Cra.13a \# 86a - 54, Bogotá D.C. (Colombia). Pbx: +571 6168122 info@kemtek.com 
través de su utilización. Las patentes se utilizan como medida de protección y compensación al inventor, proporcionándole un mercado monopolizado en un territorio durante un periodo de tiempo, usualmente de 20 años(SIC, 2011). Los primeros datos de patentes datan de la época del renacimiento en Europa; cuando a John of Utynamse otorgó en el Reino Unidouna patente por un proceso de fabricación de vidrios. Para finales del siglo XVIII, la concepción de patente aparece ampliamente difundida y a comienzos del siglo XX, EEUU y Europa poseían el 97\% de las patentes en el mundo. Tan solo 75 años después, las multinacionales japonesas lograron obtener una posición dominante en la oficina de patentes de la nación norteamericana (USPTO) (Macleod, 2006). Las Oficinas de Patentes y Propiedad Intelectual dan un gran paso hacia la armonización en el año de 1980 al establecerse la Organización Mundial de Propiedad Intelectual (WIPO) ligada a la Oficina Europea de Patentes (EPO). La USPTO, en el año de 1995, actualiza su regulación de patentes alineándose con el sistema de patentes de otros países(Reuters, 2012). Finalmente, para el año 2013, se espera el lanzamiento de la Clasificación de Patentes Cooperativa (CPC) con lo cual se pretende que la USPTO y la EPO usen el mismo sistema de clasificación de patentes(USPTO, 2011). Desde el año 2004, las oficinas de patentes y propiedad intelectual han establecido una clasificación específica para las innovaciones en nanotecnología, contando a la fecha de este artículo con más de 130 mil patentes identificadas en esta clasificación (EPO, 2012).

En general, los temas de propiedad y derechos sobre la innovación no son de común conocimiento entre los estudiantes, inventores, investigadores y administradores. ¿Sería importante entonces que quienes participan en actividades de innovación tengan el conocimiento base de propiedad intelectual y proveerlo como parte de la plataforma de entes educativos y de investigación? ¿Qué tan oportuno resulta conocerlo hasta el final del desarrollo? El presente documento recorre el estado actual de las patentes otorgadas en este campo en las principales economías globales (EEUU, Europa y Japón) y hace especial énfasis en las actividades desarrolladas en Latinoamérica (Argentina, Brasil, Chile, Colombia y México).

\section{¿QuÉ ES UNA PATENTE Y CUÁL ES SU RELACIÓN CON NANOTECNOLOGÍA?}

El uso del término "patentes" es común en la investigación, ¿pero, realmente se conoce cual es su significado y lo que implica? Existen diversas definiciones con variaciones sutiles para definir patente,dependiendo de la oficina de patentes. Sin embargo, la más utilizada es la de WIPO y se menciona a continuación:

Una patente es un derecho exclusivo concedido a una invención, es decir, un producto o procedimiento que aporta, en general, una nueva manera de hacer algo o una nueva solución técnica a un problema. Para que sea patentable, la invención debe satisfacer determinados requisitos que definen las oficinas de patentes.(WIPO, 2012a)

Las patentes son un subgrupo de la propiedad industrial que, a su vez, hace parte de lo que se conoce como propiedad intelectual. La gráfica 1 muestra los diferentes niveles para cada uno de los tipos de propiedad intelectual. Las patentes de este modo están divididas en dos grandes grupos: patentes de invención y patentes de modelos de utilidad. Las primeras requieren tres condiciones para que sea otorgada: no- 
GRÁFICA 1. Jerarquía de la propiedad intelectual. Que se encuentra a cargo las Oficinas de Patentes y Propiedad Intelectual. Las patentes pertenecen al subgrupo de Propiedad Industrial.

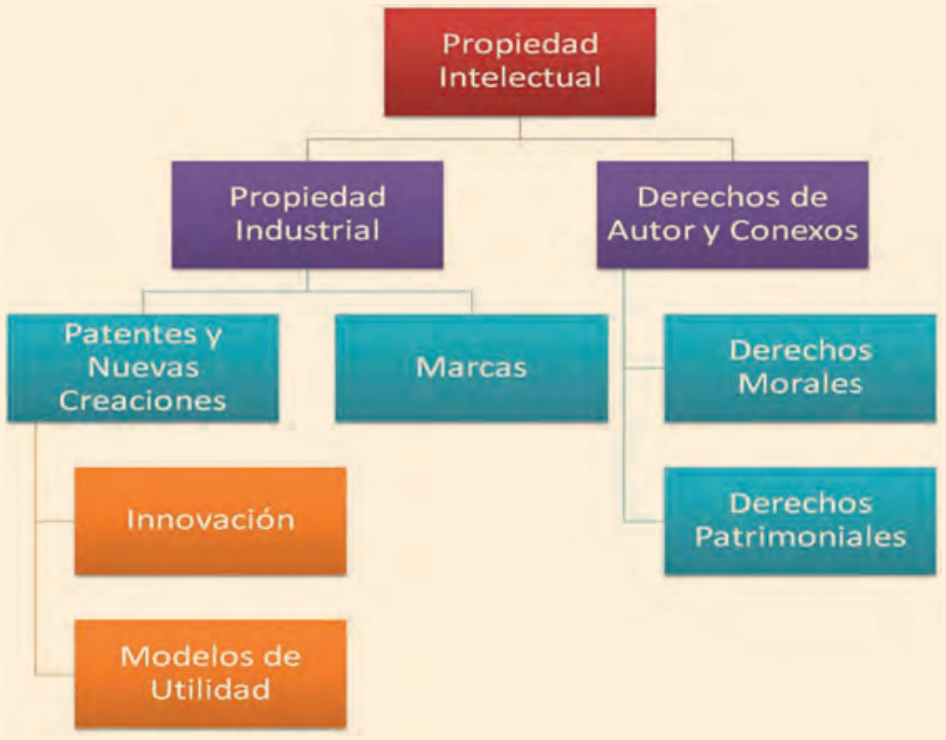

vedad, inventiva e industrializable, estas patentes tienen un periodo de protección de 20 años. Las segundas sólo brindan 10 años y deben cumplir dos condiciones: novedad e industrialización (SIC, 2011).

La aplicación de las patentes sólo tienen validez en el territorio donde fueron solicitadas. No obstante, es posible someter a través del Tratado de Cooperación en la materia de Patentes (TCP) una solicitud para cualquiera de los 146 países que hacen parte del tratado. En el cual se incluyen todos los países de Latinoamérica a excepción de Bolivia, Paraguay y Uruguay (OMPI, 2012).

Las primeras patentes asociadas con nanotecnología aparecen en 1976 en la USPTO y en 1980 para EPO y JPO (Li, Lin, Chen, y Roco, 2007). Presentando un incremento exponencial desde 1995(Dang, Zhang, Fan, Chen, y Roco, 2010). Sólo hasta el 2004 se clasifican dentro de un grupo específico en EPO y USPTO.

El número de patentes asociadas a nanotecnología ha presentado un crecimiento significativo desde finales de la década de los 90cuando existían 5,177 patentes registradas hasta mediados del 2012 con 130,780 patentes registradas, es decir, 25 veces más, gráfica 2 (Li et al., 2007)(Dang et al., 2010) (EPO, 2012). Este crecimiento se ha visto principalmente en el área de ciencias de materiales, ingeniería química y física. Durante el año de 2010 y 2011 hay un decrecimiento en el número de patentes otorgadas. Lo cual coincide con la disminución de inversión por la industria y el gobierno durante los años previos producto de la crisis económica del año 2007 al 2009 (BEA, 2012); durante el primer semestre de 2012 se registraron 5500 patentes con lo que la tendencia negativa que se presentó durante los 2 últimos años empieza a cambiar (ELSEVIER, 2012). La principal oficina de patentes que recibe solicitudes con el tér- 
GRÁFICA 2. Comportamiento del número de patentes por año que incluyen el término nano bien en su titulo o en el resumen desde 1997 hasta el 2012. Para el último año solo se tomo el primer semestre.

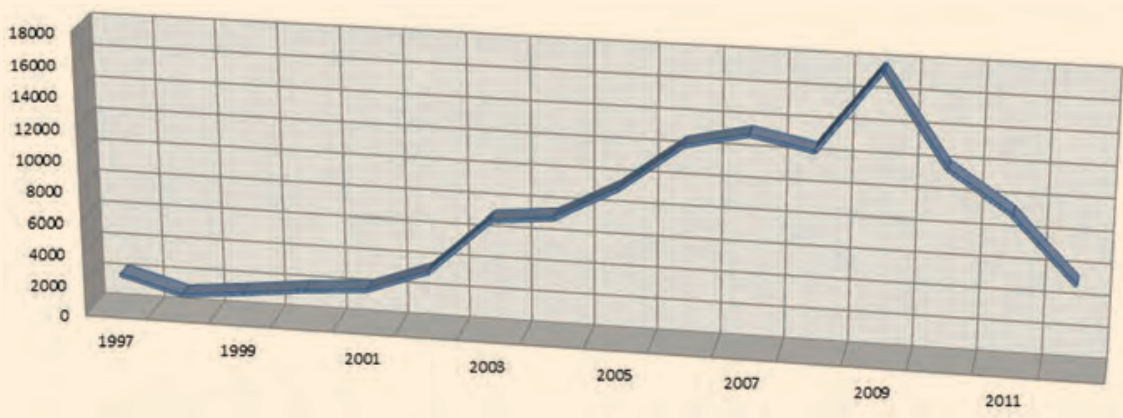

mino nano en su titulo o resumen es la oficina de patentes de Estados Unidos (USPTO) con el 56\% de todas las patentes, seguida de la oficina del Tratado de Patentes (PCT) con $28 \%$ de estas, la oficina de patentes de la Comunidad Europea (EPO) que representa 11\% y finalmente la oficina de Japón (JPO)con el 4\% (ELSEVIER, 2012; EPO, 2011; Murphy, Munshi, Kurian, Lakhtakia, \& Bartlett, 2011).

La clasificación e identificación de patentes en el ámbito de nanotecnología es un gran desafío en el proceso de clasificación cada una de las oficinas de patentes. Inicialmente, la clasificación se había asociado al tamaño de sus componentes activos o estructuras entre 1 a $100 \mathrm{~nm}$. Para USPO se usa el código 977, para EPO se uso Y01N y para JP0 era ZMN. Con esta clasificación se buscaba establecer no sólo que el titulo o el resumen hiciera referencia a nanotecnología sino que se tratase de un invento novedoso con esta tecnología (ver tabla I). A partir de 2011 la EPO y la clasificación internacional de patentes (IPC) establecieron para las patentes en nanotecnología el nuevo código B82Y; con lo cual es posible identificar las patentes para nanotecnología(EPO, 2011). Sin embargo, entre éstas dos es fácil encontrar discrepancias en el número y la forma de su clasificación.

\section{LATINOAMÉRICA Y LAS PATENTES EN NANOTECNOLOGÍA}

La existencia de patentes en la base de datos de WIPO incluyen desarrollos nanotecnológicos en Latinoamérica y sólo se encuentran aplicantes de Argentina, Brasil, Colombia, Chile y México. De las cerca de 100,000 patentes en nanotecnología que existen en el mundo, únicamente 333 se encuentran en la región. El 90\% de éstas 333 patentes están en las oficinas de Brasil y México. Lo que necesariamente evidencia un rezago importante frente al desarrollo global. Adicionalmente, el $80 \%$ de las patentes son realizadas por extranjeros, quedando un pequeño número de patentes desarrolladas por centros de investigación, universidades o empresas locales.

Cuando analizamos el tipo de aplicante si se trata de empresas, universidades $u$ otros, encontramos que la región no es uniforme en ello, en países como Colombia y Chile fundamentalmente las patentes provienen de las universidades, en países como Brasil y México prima el desarrollo de la industria y en Argentina hay un desarrollo 
TABLA I. Definiciones para nanotecnología en las principales oficinas de patentes

\begin{tabular}{|c|c|l|}
\hline Oficina & Código & \multicolumn{1}{c|}{ Definición para nano-patente } \\
\hline USPTO & 977 & $\begin{array}{l}\text { "Cubre las actividades relacionadas con la investigación y el desarrollo } \\
\text { tecnológico a los niveles atómico, molecular o macromolecular, donde } \\
\text { al menos una de sus dimensiones se encuentra en la escala de 1 a } \\
100 \mathrm{~nm} \text {. Y proporciona una comprensión fundamental de fenómenos } \\
\text { y materiales a nanoescala usando y creando estructuras, dispositivos y } \\
\text { sistemas novedosos que tengan propiedades y funciones secundarias } \\
\text { de su tamaño". }\end{array}$ \\
\hline EPO & $\begin{array}{c}\text { Y01N- } \\
\text { B82Y }\end{array}$ & $\begin{array}{l}\text { "El término nanotecnología cubre entidades con un tamaño geométri- } \\
\text { camente controlado al menos en un componente funcional inferior a } \\
100 \text { nm en una o más dimensiones susceptible de tener efectos físicos, } \\
\text { químicos o biológicos intrínsecos a su tamaño". }\end{array}$ \\
\hline
\end{tabular}

mixto entre industria y universidades. En la gráfica 3, se pueden observar estas proporciones. El área de las patentes en nanotecnología de la región esta en ciencia de materiales seguido de la investigación biomédica y química.

Se observan interacciones entre la academia e industrias de diversos países como es el caso de las Universidad Chilena de Concepción, Andromaco S.A. (Chilena) y ABL Pharma (Colombiana) filial de la primera que poseen dos patentes bajo la clasificación B82Y en EPO. En el caso de México el sector petrolero en compañía de universidades norteamericanasposee el 30\% de las patentes. Brasil tiene un importante desarrollo en la industria cosmética y farmacéutica. En el caso de Chile, el 100\% de

GRÁFICA 3. Número de patentes por cada uno de los cinco países de la región y el porcentaje de participación de las universidades como aplicantes en las mismas. Se observa cómo Brasil tiene el mayor número con una participación de universidades del 33\% mientras Chile y Colombia poseen el menor número con una participación mayor de las universidades del $80 \%$ y $60 \%$, respectivamente $(E P O, 2012)$

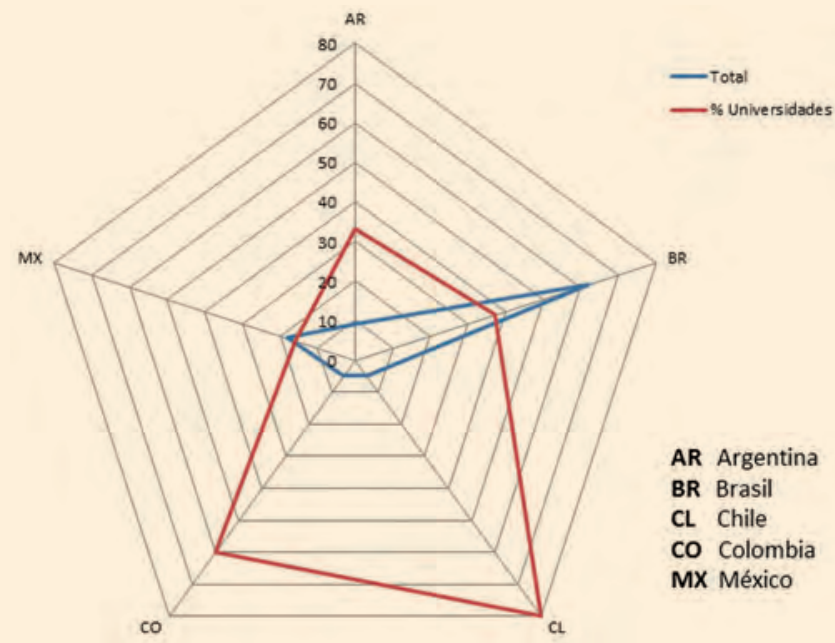


los avances en nanotecnología están asociados a universidades de las cuales el $60 \%$ son locales. Las principales áreas de investigación en la región, son ciencias de materiales, farmacéutica y cosméticos, ver gráfica 4.

GRÁFICA 4. Se presentan la principales áreas en las que la región posee patentes. Destacan ciencias de materiales y la industria farmacéutica y cosmética.

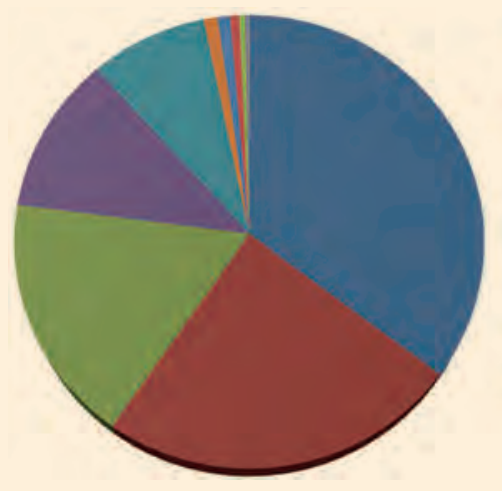

Ciencia de materiales

Farmacéutica

Física

Ingeniería

Bioquímica, genérica, biología molecular

Desde Latinoamérica las principales oficinas de patentes a las cuales se solicita patentes son: WIPO con el 36\%, USPTO con el 24\% y EPO con el 11\%. Otras oficinas como las de México y Colombia reciben solicitudes desde aplicantes brasileros para proteger sus creaciones en estos países. En la gráfica 5, se puede observar dicho comportamiento. Cabe aclarar que la solicitud de patentes de inventores en su mismo territorio en Latinoamérica es muy escasa llegando sólo al 12\% en el caso de Brasil que

GRÁFICA 5. Distribución de las oficinas de patentes donde los inventores latinoamericanos suelen solicitar protección por patentes de su invención (WIPO, 2012).

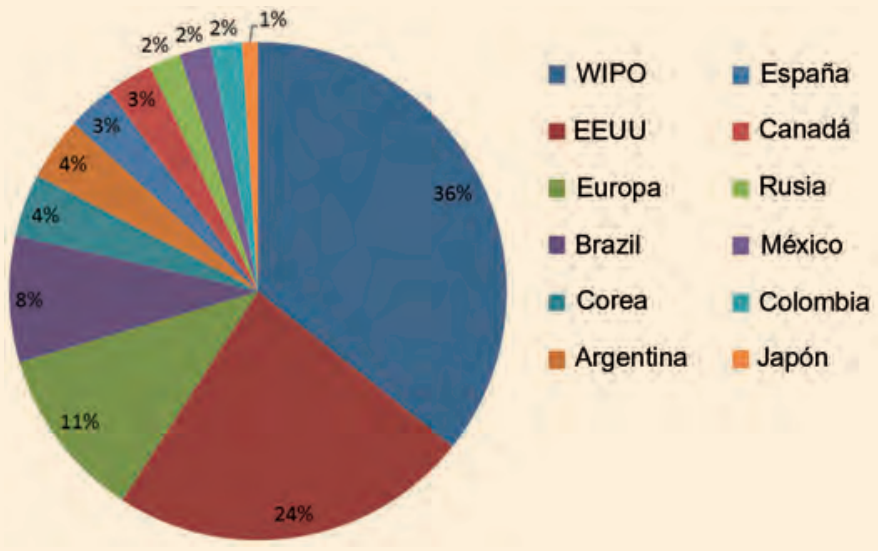


es el que más posee. Esta distribución responde al interés de utilizar la patente en los mercados de mayor demanda y así favorecer la explotación de la patente. Sin embargo, competir allí resulta mucho más costoso por lo cual se requiere la inversión de capital por un tercero que permita financiar la producción y comercialización.

En cada territorio la oficina de patentes establece los procedimientos y estatutos para la solicitud de patentes dentro de los lineamientos del Tratado de Cooperación de Patentes PCT. La tabla II, relaciona las oficinas de patentes de cada uno de los cinco países y el número de patentes concedidas que incluyen el término nano en sus territorios.

TABLA II. Oficina de Patentes por país con su website y el número de patentes concedidas en los territorios

\begin{tabular}{|c|l|l|c|}
\hline País & \multicolumn{1}{|c|}{ Oficina } & Website & Patentes concedidas \\
\hline $\mathbf{M X}$ & $\begin{array}{l}\text { Instituto Mexicano de la } \\
\text { Propiedad Industrial }\end{array}$ & http://www.impi.gob.mx & 28 \\
\hline $\mathbf{A R}$ & $\begin{array}{l}\text { Instituto Nacional de la } \\
\text { Propiedad Industrial }\end{array}$ & http://www.inpi.gov.ar & 13 \\
\hline $\mathbf{B R}$ & $\begin{array}{l}\text { Instituto Nacional de } \\
\text { propiedad Industrial Ministerio } \\
\text { de Desarrollo, Industria y } \\
\text { Comercio Internacional }\end{array}$ & http://www.inpi.gov.br & 4 \\
\hline $\mathbf{C L}$ & $\begin{array}{l}\text { Instituto Nacional de } \\
\text { propiedad Industrial Ministerio } \\
\text { de Economía }\end{array}$ & http://www.inapi.cl/ & 5 \\
\hline CO & $\begin{array}{l}\text { Superintendencia de Industria } \\
\text { y Comercio Ministerio de } \\
\text { Industria, Comercio y Turismo }\end{array}$ & http://www.sic.gov.co & 4 \\
\hline
\end{tabular}

A continuación se describe el proceso para la obtención de patentes en el territorio colombiano, el cual no varía sustancialmente de los otros países. La gráfica 6 describe los pasos a seguir.

\section{Procedimiento de patentes en Colombia}

En Colombia la Oficina de Patentes se encuentra administrada por la Superintendencia de Industria y Comercio (SIC), esta oficina se encarga de otorgar las patentes para el territorio Colombiano. El proceso puede llevar de 3 a 5 años dependiendo de la complejidad de la solicitud. Éste está dividido en tres fases: la primera es la fase de Radicación y Admisión del trámite, lo fundamental en ella es la presentación completa de los documentos y el pago del examen de forma; si pasa esta etapa sigue la fase de publicación (aprox. 18 meses) donde terceros pueden oponerse; a continuación se realiza la petición de examen de patentabilidad (hasta 6 meses) y el examen de fondo donde se concede o niega la patente. Adicionalmente, se debe realizar un 
pago por mantenimiento de la patente de forma anual. Una vez otorgada la patente se tienen tres años para iniciar la producción del desarrollo tecnológico o de lo contrario el Estado está en la capacidad de otorgar licencias a terceros para que la exploten, ver gráfica 6.

GRÁFICA 6. Pasos para la obtención de una patente en Colombia a través de la SIC (SIC, 2011).

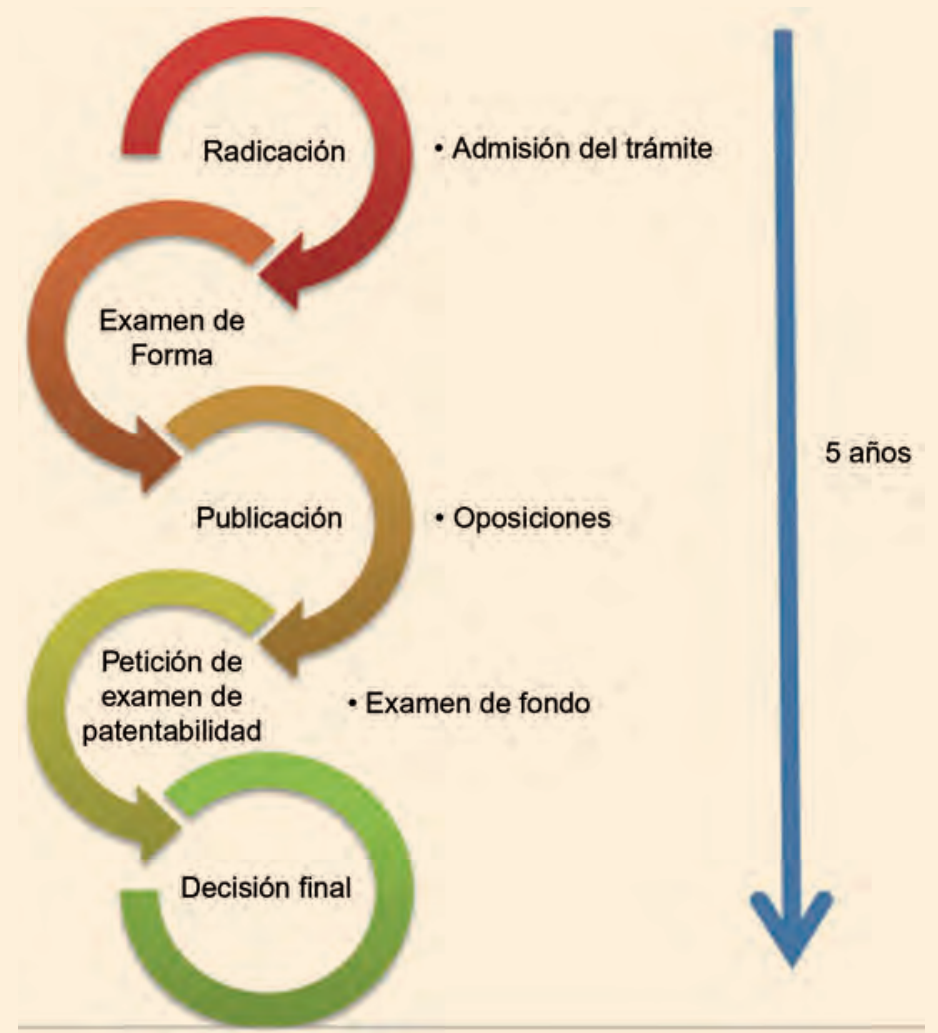

Las universidades y las patentes

En el marco de investigación colombiana existe una brecha importante entre los mismos grupos de investigación, la industria y el gobierno con el propósito de promover el desarrollo productivo de los proyectos de investigación que culminen en aplicaciones útiles para la sociedad que estimulen el crecimiento económico basado en nanotecnología. Es así como en la base de datos de GrupLAC de ScienTI cerca de 106 grupos de investigación se encuentran inscritos con proyectos relacionados con nanotecnología (SienTI, 2012). Sin embargo, sólo tres universidades colombianas tie- 
nen patentes en nanotecnología, ${ }^{1}$ en las áreas de bioquímica e ingeniería química. No obstante, estas patentes no poseen un buen nivel de citación evidenciando un menor impacto en la sociedad(SIC, 2011).

\section{Patentes en nanotecnología en Colombia (casos exitosos)}

Lograr que los procesos de investigación y desarrollos empresariales brinden provecho a la sociedad es uno de los retos más grandes cuando se encuentra en investigación aplicada. Un ejemplo de esto es el trabajo realizado por la compañía KEMTEK®. Desde principios de los años 80 empezó a investigar sobre los beneficios que los sistemas coloidades naturales podrían brindar a la industria de alimentos y a la agricultura, llegando a desarrollar con Nanotecnología Aplicada Sistemas Coloidales diseñados a la medida (Tailor-made) para el transporte de principios o ingredientes activos mediante nanoglóbulos; maximizando la efectividad de fertilizantes y plaguicidas, mejorando los rendimientos para los agricultores en los diferentes cultivos y reduciendo los costos al igual que la presencia de residuos químicos en el medio ambiente.

Las especialidades de STC@ - Sistemas de Transporte Coloidal $®$ - se han aplicado en diferentes sectores de la industria. El desarrollo de productos especializados como KEM-KOL $®$ ha sido de gran impacto en la agricultura siendo utilizado para fertilizantes, insecticidas, herbicidas, fungicidas y hasta bioplaguicidas. Otro sector de alto impacto es el de la construcción con el producto STASOIL®; el cual es utilizado en la construcción de carreteras, en estabilización de bases y subases, permitiendo reducir espesores de diseño de capas a tratar en más de un 50\% comparado con espesores a tratar en sistemas tradicionales de diseño de vías. Se reduce drásticamente espesor en diseño de capas de pavimento, ya sea, asfalto o concreto. En el campo de la generación de energía, KEMTEK® ha desarrollado especialidades de STC $®$ para fluidificación y recuperación secundaria de crudos pesados en la industria petrolera, reducción y reciclaje de agua, mejoras de combustión en equipos generadores de electricidad reduciendo emisión de particulado en más de $60 \%$ e incrementando eficiencia en generación eléctrica.

El éxito en un desarrollo industrial está asociado con lograr expansión comercial por sus resultados innovadores, benéficos y la difusión del conocimiento para la comunidad, sin el riesgo de perder su propiedad intelectual por violación de terceros de los mecanismos de protección. Durante las décadas de trabajo y dedicación con las actividades de protección intelectual KEMTEK® ha encontrado desgastante proteger y mantener propiedad intelectual contra la competencia desleal y facilista. La falta de periodismo verdaderamente científico y no comercial facilita el posicionamiento de la "ignorancia creativa comercial" que desvirtúa el trabajo científico y tecnológico.

\section{Conclusiones}

La obtención de beneficios para la sociedad de las actividades de investigación aplicadas se ven reflejadas en la obtención de productos que lleguen a las personas y brin-

\footnotetext{
1 Universidad del Norte, Universidad Javeriana y Universidad de Antioquia.
} 
den soluciones a sus problemas. La nanotecnología se convierte en una importante oportunidad para manipular la materia e innovar, resultados que se están observando en diferentes lugares del mundo.

Se evidencia en la región existen dificultades en la construcción de políticas eficientes que permitan a los centros de investigación culminar sus trabajos en exitosas actividades del sector productivo. Llevar a cabo actividades de integración entre los organismos del Estado, inversionistas, emprendedores, industria y centros de investigación para dirigir esfuerzos a las necesidades y brindar alternativas de éxito a nuestra comunidad. La desproporción entre el número de centros de investigación en Colombia y el número de patentes asociadas a nanotecnología nos hace reflexionar sobre esta brecha.

Se presenta en este documento una revisión del estado actual de las patentes esperando que sobre esta discusión sea posible construir en la región conciencia de la importancia de involucrar a los diferentes actores en la necesidad de incluir en sus planes las etapas de protección intelectual.

¿Se constituye en un deber del investigador conocer sobe propiedad intelectual? ¿A la par con la preparación para la investigación debería por parte de las universidades incluir catedras de propiedad intelectual? ¿Estamos dirigiendo adecuadamente nuestros recursos y esfuerzos para alternativas que nos brinden mejores oportunidades competitivas como país hacia el futuro?

\section{BIBLIOGRAFIA}

BEA. (2012). Bureau of Economic Analysis. Retrieved 19 Sep 2012 <http://www.bea.gov/ itable/index.cfm>.

Dang, Y., Zhang, Y., Fan, L., Chen, H., \& Roco, M. (2010). Trends in worldwide nanotechnology patent applications: 1991 to 2008. Journal of Nanoparticle Research, 12(3), 687706. doi: 10.1007/s11051-009-9831-7

ELSEVIER. (2012). SciVerse - HUB - Search Results. Available from ELSEVIER SciVerse-HUB <http://www.hub.sciverse.com/action/search/results?st=nano\%2C+patent\&clust ers=srctype $\% 2 \mathrm{Clup} \% 2 \mathrm{C} 0 \% 2 \mathrm{Clwp} \% 2 \mathrm{C} 0 \% 2 \mathrm{Clep} \% 2 \mathrm{C} 0 \% 2 \mathrm{Cljp} \% 2 \mathrm{C} 0 \% 2 \mathrm{Clgp} \% 2 \mathrm{C} 0 \%$ 3Apat\%5Blup\%2Clwp\%2Clep\%2Cljp\%2Clgp\%5D>.

EPO. (2011). Nanotechnology and Patents. Munich: Retrieved from <http://www.epo.org/ service-support/publications/issues/nanotechnology.html>.

EPO. (2012). Espacenet Patent search. Available from EPO Worldwide Database 5.7.38; 93p Retrieved 19 Sep 2012, from European Patent Office <http://worldwide.espacenet.com/advancedSearch?locale=en_EP>.

Li, X., Lin, Y., Chen, H., \& Roco, M. (2007). Worldwide nanotechnology development: a comparative study of USPTO, EPO, and JPO patents (1976-2004). Journal of Nanoparticle Research, 9(6), 977-1002. doi: 10.1007/s11051-007-9273-z

Macleod, C. (2006). untitled. The British Journal for the History of Science, 39(3), 438-439.

Murphy, P., Munshi, D., Kurian, P. A., Lakhtakia, A., \& Bartlett, R. V. (2011). 5.15 - Nanotechnology, Society, and Environment. In L. A. Editors-in-Chief: David, D. S. Gregory \& P. W. Gary (Eds.), Comprehensive Nanoscience and Technology (pp. 443-476). Amsterdam: Academic Press. 
OMPI. (2012). Tratados administrados por la OMPI. Miembros de los órganos de la OMPI.Retrieved Sep 20 2012, 2012, from <http://www.wipo.int/treaties/es/ShowResults. jsp?lang=es\&search_what=B\&bo_id=13>.

Reuters, T. (2012). The History of Patents - IP \& Science - Thomson Reuters.

SIC. (2011). ABC de la Propiedad Industrial. Bogota D.C.: Superintendencia de Industria y Comercio.

SienTI, C. (2012). GrupLAC. Retrieved 19 Sep 2012<http://201.234.78.173:8083/cienciawar/>.

USPTO, E. (2011). Cooperative Patent Classification. <www.cpcinfo.org: Retrieved from http://www.cooperativepatentclassification.org/publications.html>.

WIPO. (2012a). Patents. Retrieved 19 Sep 2012, 2012, from <http://www.wipo.int/patentscope/en/>.

WIPO. (2012b). WIPO IP Facts and Figures 2012. In W.-W. I. P. Organization (Ed.), WIPO Economics \& Statistics Series (Vol. 2012, pp. 48). Geneva: WIPO. 